\title{
Assessing The Inflammatory Severity of The Terminal lleum In Crohn Disease Using Radiomics Based On MRI
}

Honglei Ding

The First Affiliated Hospital of Zhejiang Chinese Medical University

Jiaying Li

The First Affiliated Hospital of Zhejiang Chinese Medical University

Kefang Jiang

The First Affiliated Hospital of Zhejiang Chinese Medical University

Chen Gao

The First Affiliated Hospital of Zhejiang Chinese Medical University

\section{Liangji Lu}

The Second Affiliated Hospital of Zhejiang University School of Medicine

Huani Zhang

The First Affiliated Hospital of Zhejiang Chinese Medical University

Kefeng Zhou

The First Affiliated Hospital of Zhejiang Chinese Medical University

Haibo Chen

The First Affiliated Hospital of Zhejiang Chinese Medical University

\section{Xuning Gao}

The First Affiliated Hospital of Zhejiang Chinese Medical University

Zhichao Sun ( $\nabla$ sunzhichao@zcmu.edu.cn )

The First Affiliated Hospital of Zhejiang Chinese Medical University

\section{Research Article}

Keywords: Crohn Disease, Terminal ileum, Radiomics, Magnetic resonance Imaging

Posted Date: January 5th, 2022

DOl: https://doi.org/10.21203/rs.3.rs-1205199/v1

License: (c) (i) This work is licensed under a Creative Commons Attribution 4.0 International License. Read Full License 


\section{Abstract}

Background: Evaluating inflammatory severity using imaging is essential for Crohn's disease (CD), but it is limited by potential interobserver variation and subjectivity. We compared the efficiency of magnetic resonance index of activity (MaRIA) collected by radiologists and a radiomics model in assessing the inflammatory severity of terminal ileum (TI).

Methods: 121 patients were collected from two centers. Patients were divided into ulcerative group and mucosal remission group based on the TI Crohn's disease Endoscopic Severity Index (tCDEIS). The consistency of bowel wall thickness (BWT), relative contrast enhancement (RCE), edema, ulcer, MaRIA and features of the region of interest (ROI) between radiologists were described by weighted $k$ coefficient and intraclass correlation coefficient (ICC), and developed receiver operating curve (ROC) of MaRIA. The radiomics model was established using reproducible features of logistic regression based on arterial staging of T1WI sequences. Delong test was used to compare radiomics with MaRIA.

Results: The consistency between radiologists were moderate in BWT (ICC $=0.638)$, fair in edema $(k=0.541)$, RCE (ICC=0.461), MaRIA (ICC=0.579) and poor in ulcer $(\mathrm{k}=0.271)$. Radiomics model was developed by 6 reproducible features (ICC $=0.93-0.96)$ and equivalent to MaRIA which evaluated by the senior radiologist $(0.872$ vs 0.883 in training group, 0.824 vs 0.783 in testing group, $P=0.847,0.471$ ), both of which were significant higher than MaRIA evaluated by junior radiologist(AUC: 0.621 in training group, 0.557 in testing group, all, $P B 0.05$ ).

\section{Introduction}

Crohn's Disease (CD) is a chronic inflammatory bowel disease with unknown etiology [1]. CD presents with periods of clinical remission and activity, persistent inflammatory activity, and is thought to trigger intestinal damage, leading to complications and affecting overall treatment [2-3]. The terminal ileum( $\mathrm{TI})$ is the most common site of $\mathrm{CD}$, and previous studies have shown complications such as stenosis are common when involved[4]. Although the TI can be reached by conventional colonoscopy, complete information cannot be obtained, the use of small bowel endoscopy may compensate for this shortcoming, but complications or side effects need to be considered[5-8].

Magnetic resonance imaging (MRI) can non-invasively assess the small bowel environment and reveal the extent of extraluminal disease, therefore, it has been more frequently used than endoscopy in the clinical management of $C D$ [9-10]. The magnetic resonance index of activity (MaRIA), which was developed to standardize the MRI findings, has been shown to be excellent in detecting the inflammation of $C D$ with the CDEIS as a reference standard [11-12].

However, MaRIA results are subjective and depend on clinician experience[13]. Therefore, an accurate, reproducible and objective assessment for $\mathrm{CD}$ severity is of particular importance. In recent years, radiomics has been developed as a new method for reflecting the changes of diseases by mining non-visual data of images and widely validated in the field of cancer imaging [14-16]. The high-dimensional features of images may accurately and objectively reflect the manifestations of lesions. Therefore, this study aimed to use radiomics in detecting CD inflammatory severity and compare the MaRIA evaluated by different experienced radiologists in order to quantify the efficacy of radiomics.

\section{Methods And Materials}

Subject Selection 
The ethics committee of our institutional approved this retrospective study(2020-KL-035-01) and the need for written informed consent was waived. Patients diagnosed with CD in two centers were retrospectively collected. All subjects were screened according to the inclusion and exclusion criteria. The inclusion criteria were as follows: (1) patients who performed ileocolonoscopy; (2) patients who underwent MRI scan. The exclusion criteria included: (1) Incomplete clinical data; (2) The interval between endoscopy and MRI was more than 3 days, in order to avoid the change of intestinal real-time state; (3) The area of interest (ROI) cannot be delineated or the TI is not clearly visible on imaging; (4) Patients whose TI was dominated by fibrous stenosis or surgically removed; (5) L2 sub-type and upper digestive tract involvement with L2 were excluded according to the Montreal Classification because of uninvolved $\mathrm{TI}$.

\section{Mri Acquisition}

All patients in two centers were imaged using a 3.0T MR (General Electric Company, USA). Patients were placed in the supine position on the examination bed. An abdominal surface coil was used to modulate the signal. Before imaging, patients were given $20 \mathrm{mg}$ Raceanisodamine Hydrochloride intramuscularly to reduce bowel peristalsis and dilate the lumen fully. The scanning sequences mainly included T2-weighted fat-suppression sequences, diffusion-weighted imaging sequences (b-values included 500 and $800 \mathrm{~mm}^{2} / \mathrm{s}$ ), and contrasted enhancement based on liver acquisition with volume acceleration (LAVA) sequence. Arterial-phase enhanced imaging based on liver acquisition with volume acceleration (LAVA) sequence was acquired $20 \mathrm{~s}$ after intravenous administration of $0.1 \mathrm{mmol} / \mathrm{kg}$ body weight of Gadolinium-DTPA through the cubital vein at a rate of $2.5 \mathrm{~mL} / \mathrm{s}$. The detailed parameters are listed in Table1. Due to the application of the arterial phase in processing of radiomics, the scanning parameters of contrasted sequence in center 2 was only presented.

Table 1

Protocol for MR image acquisition

\begin{tabular}{|c|c|c|c|c|c|c|c|}
\hline & Plane & $\mathrm{TR} / \mathrm{ms}$ & $\mathrm{TE} / \mathrm{ms}$ & $\begin{array}{l}\text { Slice } \\
\text { thickness/mm }\end{array}$ & FOV & Matrix & NEX \\
\hline $\mathrm{T} 1 \mathrm{WI}+\mathrm{C}($ Center 1$)$ & Axial/coronal & 3.7 & 1.1 & 5 & $280 \times 80$ & $288 \times 288$ & 1 \\
\hline $\mathrm{T} 1 \mathrm{WI}+\mathrm{C}($ Center 2) & Axial & 3.7 & 1.6 & 5 & $280 \times 80$ & - & 1 \\
\hline T2WI-FS(Center 1) & Axial & 3333.3 & 85.2 & 7 & $36 \times 80$ & - & 2 \\
\hline $\begin{array}{l}\text { DWI }\left(500 / 800 \mathrm{~mm}^{2} / \mathrm{s} \text {, Center }\right. \\
\text { 1) }\end{array}$ & Axial & 7058.8 & 81.8 & 7 & $36 \times 80$ & - & 4 \\
\hline \multicolumn{8}{|l|}{ Note: TR: repetition time } \\
\hline \multicolumn{8}{|l|}{ TE: echo time } \\
\hline \multicolumn{8}{|l|}{ FOV: field of view } \\
\hline \multicolumn{8}{|l|}{ NEX: number of excitations } \\
\hline \multicolumn{8}{|c|}{ T1WI+C: Contrasted T1-weighted image } \\
\hline \multicolumn{8}{|c|}{ T2WI-FS: T2-weighted image-Fat Suppression } \\
\hline DWI: Diffusion weighted ima & & & & & & & \\
\hline
\end{tabular}




\section{Endoscopic Data And Maria Collection}

Ileocolonoscopy was used as a reference standard of inflammatory bowel disease. All patients were given 3000-4000 $\mathrm{mL}$ of compound polyethylene glycol and electrolyte solution for intestinal cleansing the night before their examination, and to ensure the intestinal tract was clearly visible under endoscopy. $40 \mathrm{ml}$ Simethicone Emusion was also given orally on the morning of inspection. For comparing the consistency of the MaRIA and endoscopic assessment of the ROI, the $\mathrm{Tl}$, which was defined as the segment of the small intestine within $10 \mathrm{~cm}$ of the ileocecum, was assessed by CDEIS of terminal ileum(tCDEIS) rather than the overall CDEIS score. For CDEIS calculation, the endoscopic variables were as originally defined: deep ulcers and superficial ulcers (presence or absence), ulcerated surface and affected surface (evaluated on a $10 \mathrm{~cm}$ linear analogue scale), ulcerated and nonulcerated stenosis [17]. All procedures were carried out by a gastroenterologist with more than 20 years of experience using the standard equipment (CFQ240, Olympus, Japan).

MaRIA was evaluated by MR findings including bowel thickness, relative contrast enhancement (RCE=((WSI postgadolinium - WSI pregadolinium) / (WSI pregadolinium) $\times 100 \times($ s.d. noise pregadolinium / s.d. noise postgadolinium))), edema (hyperintensity on T2-weighted sequence relative to the signal of the psoas muscle) and ulcers (defined as deep depressions in the mucosal surface) [10]. Two radiologists, one with 15 years of experience in abdominal imaging (ER1) and one with 5 years of experience in abdominal imaging (ER2) were asked to calculate the score in the thickest region of the bowel wall according to these following formulas: MaRIA $=1.5 \times$ bowel thickness + $0.02 \times \mathrm{RCE}+5 \times$ edema $+10 \times$ ulcer. The two radiologists were blind with each other and the endoscopic results.

MaRIA less than 7 indicates normal mucosa, a score of 7-11 is considered mild disease, a score greater than 11 indicates ulcerated lesions[11], and MaRIA less than 11 was treated as ulcer healing. In CDEIS score, 3.5 and below are classified as mild inflammation with a tendency toward mucosal healing, a score of 3.5-7 is considered moderated disease and less than 7 was treated as endoscopic remission, a score more than 7 was treated as ulcerative disease [18], which indicated a poorer prognosis and moderate-high risk of clinical treatment [19]. Therefore, all patients were classified as ulcerative group(UG, tCDEIS > 7, MaRIA > 11), and mucosal remission group (MG, tCDEIS $\leq 7$, MaRIA $\leq 11)$.

\section{Radiomics Processing}

The arterial stage of T1 weighted enhanced imaging of all patients was imported into ITK-SNAP (Version 3.80) software in DICOM format. Two radiologists who evaluated MaRIA performed outlining along the TI wall on the axial image slice by slice manually. The lumen of intestinal was excluded and the intestinal wall was not clearly displayed or the visual overlap of adjacent intestinal walls were not included in the ROI. Part of the outlining results were shown in Figure 2A and Figure 2B.

All the outlining images and the corresponding primary images were imported into Dr. Wise Multimodel Research Platform (Version1.6.3.6 Deepwise\&League of PHD Technology Co., Ltd, Beijing, China) for one-to-one matching, labeling and ROI features extraction. 1648 features were selected, including shape feature, Gray Level Co-occurrence Matrix (GLCM), Gray Level Run Length Matrix (GLRLM), Gray Level SizeZone Matrix (GLSZM), Gray Scale Correlation Gray Level Dependence Matrix (GLDM) and Neighborhood Gray-Tone Difference matrix (NGTDM), all these features were transformed according to LoG, Square, Square Root, Logarithm, Gradient, Wavelet and local binary pattern(LBP 2 dimension and 3dimension). All patients in center 1 were randomly divided into the training group and the experimental group in a ratio of 7:3 and underwent 5 cycles. Before feature dimension reduction, the features with a 
miss rate greater than $10 \%$ was selected to be cleared, and a correlation coefficient greater than 0.9 between features should be removed to avoid redundancy. Mutual information(MI) method was selected to control the multivariate and logistic model was selected for machine learning. The workflow in summary can be seen in Figure 2 . The patients in center 2 was used to validate the model.

\section{Calibration And Clinical Utility Of Radiomics Model}

Calibration curve was used to measure how well a probabilistic prediction of an event matches the true underlying probability of the event. Decision curve analysis was used to measure the clinical efficacy of Radiomics model; $A$ decision analysis measure, called the net benefit of the model, was calculated for the possible threshold probabilities. The benefits (proportion of true positives) and disadvantages (proportion of false positives) are added, and the diagnosis is weighted by the relative harm of false positives and false negative results. The net benefit values of the diagnostic model was standardized for prevalence.

\section{Statistical Analysis}

All statistical analyses were performed using SPSS software(version 22.0) and MedCalc software (version 15.2). Descriptive statistics were performed for part of the patient clinical data. Median(interquartile range) was performed to describe the distribution of data, such as tCDEIS, BWT, RCE, and MaRIA. The intraclass correlation coefficient(ICC) test and weighted Kappa coefficient were provided to test the consistency in measurements and features of ROI between radiologists. Area under the receiver operating curve (AUC), sensitivity and specificity were reported for presenting effectiveness of MaRIA evluated by radiologists, Delong test used to measure statistical differences between MaRIA and radiomics. A p-value less than 0.05 was considered statistically significant. Reliability was constitute "poor," "fair," "moderate," and "good" reliability, with a corresponding cutoff value of ICCs or k value of <0.4, $0.41-0.6,0.61-0.8$, and $>0.8$, and higher than 0.9 was considered the good agreement in ROI.

\section{Results}

\section{Patient Characteristics}

A total of 200 patients(141 between January 1, 2016 and April 30, 2020 from Center 1, 59 between May 1, 2019 and July 1, 2021 from Center 2) were studied and 121 patients(91 in Center 1, 30 in Center 2) who meet these requirements were evaluated finally, the patient excluded workflow can be seen in Figure 1. 68 patients(52 in Center 1, 16 in Center 2) were classified as the UG and tCDEIS in Center 1 was 10,00(IQR 2.50), Center 2 was 9.00(IQR 2.00). 53 patients (39 in Center 1, 14 in Center 2) were grouped in MG, the tCDEIS in in Center 1 was 3.50(IQR 1.50) and 3.25(IQR 2.00) in Center 2. CRP and ESR were elevated relative to reference values (normal $\leq 8 \mathrm{mg} / \mathrm{l}, \leq 7.2 \mathrm{~mm} / \mathrm{L}$ ) in 36 and 33 patients, respectively. There was significant difference in gender, and CRP, but no significant difference in average age, surgery, the history of perianal involvement and ESR in two groups (Table2). 
Table 2

Clinical and biological characteristics

\begin{tabular}{|c|c|c|c|}
\hline & UG & MG & $P$ \\
\hline Number & 68 & 53 & \\
\hline Average age/years, (Mean \pm SD) & $40.2 \pm 16.2$ & $34.1 \pm 13.6$ & 0.07 \\
\hline Female, n (\%) & $13(19.1 \%)$ & $26(49.1 \%)$ & cript $>0.01$ \\
\hline History of Perianal involvement, $\mathrm{n}(\%)$ & $15(22.1 \%)$ & $15(28.3 \%)$ & 0.43 \\
\hline History of surgery, n (\%) & $20(33.3 \%)$ & $15(32.4 \%)$ & 0.89 \\
\hline \multicolumn{4}{|l|}{ Inflammatory biomarkers, n (\%) } \\
\hline $\mathrm{ESR}(>7.2 \mathrm{~mm} / \mathrm{L})$ & $13(25.0 \%)$ & $18(45.9 \%)$ & 0.06 \\
\hline $\mathrm{CRP}(>8 \mathrm{mg} / \mathrm{L})$ & $14(22.9 \%)$ & $21(54.1 \%)$ & 0.02 \\
\hline \multicolumn{4}{|l|}{ Treatment measures, n (\%) } \\
\hline Anti-TNF antibodies & $25(20.7 \%)$ & & \\
\hline Immunosuppressant & $27(22.3 \%)$ & & \\
\hline Hormone steroid & $4(3.3 \%)$ & & \\
\hline Combination of two or more drugs & $29(24.0 \%)$ & & \\
\hline tCDEIS(IQR, Center 1$)$ & $10,00(2.50)$ & $3.50(1.50)$ & - \\
\hline tCDEIS(IQR, Center2) & $9.50(2.00)$ & $3.25(2.00)$ & - \\
\hline \multicolumn{4}{|l|}{ Note: UG: Ulcerative group } \\
\hline \multicolumn{4}{|l|}{ MG: Mucosal remission group } \\
\hline \multicolumn{4}{|l|}{ ESR: Erythrocyte sedimentation rate } \\
\hline \multicolumn{4}{|l|}{ CRP: C-reactive protein } \\
\hline tCDEIS: Crohn's Disease Endoscopic In & of Severit & terminal $\mathrm{i}$ & \\
\hline
\end{tabular}

\section{Maria And Relative Parameters Measurement}

Since the non-contrast sequence was not performed in Center 2, the MaRIA and related parameters of Center 2 were not calculated. The median value of radiologists in detecting BWT was $4.35 \mathrm{~mm}$ (IQR 1.52), $3.52 \mathrm{~mm}$ (IQR 1.39), respectively. The measurements of RCE between observers were 128.00 (IQR 75.38) and 70.00 (IQR 52.75), respectively. A moderate consistency of BWT between radiologists were detected (ICC=0.638 Table 3), fair consistency was detected in parameters of RCE, edema and ulcers reading (ICC $=0.461, k=0.541$, respectively, Table 3) and poor in ulcer $(\mathrm{k}=0.271)$ between radiologists. 
Table 3

Measurements between Radiologists and Retained Features

\begin{tabular}{|c|c|c|c|}
\hline \multicolumn{4}{|c|}{ Measurements between Radiologists } \\
\hline & ER1 & ER2 & $\mathrm{ICC} / \mathrm{k}$ \\
\hline BWT(Median, IQR) & $4.351(1.52)$ & $3.52(1.39)$ & 0.638 \\
\hline RCE(Median, IQR) & $128.00(75.38)$ & $70.00(52.75)$ & 0.461 \\
\hline MaRIA(Median, IQR) & $9.50(4.61)$ & 7.12(3.59) & 0.579 \\
\hline Edema, n (\%) & $11(12.1)$ & $4(4.4)$ & $0.541^{*}$ \\
\hline Ulcer, n (\%) & $6(6.6)$ & $1(1.1)$ & $0.271^{\star}$ \\
\hline \multicolumn{3}{|l|}{ Retained Features } & Coefficient \\
\hline \multicolumn{3}{|c|}{ Wavelet-LHL_firstoeder_Maximum } & -0.6136 \\
\hline \multicolumn{3}{|c|}{ Wavelet-LLL_firstorder_Minimum } & 0.5292 \\
\hline \multicolumn{3}{|c|}{ Wavelet-HHL_firstorder_Skewness } & 0.0128 \\
\hline \multicolumn{3}{|c|}{ Wavelet-HHH_gldm_DependenceVariance } & -0.0623 \\
\hline \multicolumn{3}{|c|}{ Wavelet-LLL_glrlm_RunVariance } & 0.3030 \\
\hline \multicolumn{3}{|c|}{ Log-sigma-2-0-mm-3D_SmallAreaEmphasis } & -0.3754 \\
\hline \multicolumn{4}{|c|}{ Noe: ER1: experienced radiologist 1} \\
\hline \multicolumn{4}{|c|}{ ER2: experienced radiologist 2} \\
\hline \multicolumn{4}{|c|}{ RCE: relative contrast enhancement } \\
\hline \multicolumn{4}{|c|}{ MaRIA: magnetic resonance index of activity } \\
\hline \multicolumn{4}{|c|}{ IQR: Inter quartile range } \\
\hline \multicolumn{4}{|c|}{ GLDM: Gray Level Dependence Matrix } \\
\hline \multicolumn{4}{|c|}{ GLRLM: Gray Level Run Length Matrix } \\
\hline \multicolumn{4}{|c|}{ ICC: intraclass correlation coefficient } \\
\hline *: Weighted Kappa cc & ficient & & \\
\hline
\end{tabular}

MaRIA score measurement between investigators was 9.50 (IQR 4.61) and 7.12 (IQR 3,59). Obviously, fair agreement was detected between two radiologists (ICC=0.579, Table 3).

\section{Features selected and retained}

An ICC higher than 0.9 was considered to be retained for dimensionality reduction. After screening, a total of 1229 features were considered to have high consistency. 5 basic clinical information features were removed and 1224 were retained for reduction. 6 highly consistent parameters (ICC:0.93-0.96, Table 3) were obtained after dimensionality reduction by $\mathrm{MI}$ method eventually.

The Radscore was calculated by the following formula: 
-0.6136 x Wavelet-LHL_firstoeder_Maximum

+ $0.5292 \times$ Wavelet-LLL_firstorder_Minimum

+ $0.0128 \times$ Wavelet-HHL_firstorder_Skewness

- 0.0623 x Wavelet-HHH_gldm_DependenceVariance

+ $0.3030 \times$ Wavelet-LLL_glrlm_RunVariance

- 0.3754 x Log-sigma-2-0-mm-3D_SmallAreaEmphasis

$+0.1135$

\section{Models For Identifying Inflammatory Severity}

The MaRIA was modeled to represent the radiologists' assessment of inflammatory severity based on MR findings. When evaluating the MaRIA acquired by ER1, the AUC was 0.883 , with a sensitivity and specificity of 0.857 and 0.909 in the training group, respectively. The AUC, sensitivity and specificity of the testing group were $0.783,0.700$, and 0.867 , separately. The model performance of ER2 had an AUC of 0.621 , with a sensitivity and specificity of 0.685 and 0.577 in the training group, respectively. The AUC in the testing group was 0.557 , the sensitivity and specificity was 0.548 and 0.603 , separately.

The radiomics model has been established to distinguish the state of ROI depending on the reserved features. In training group, the AUC, sensitivity and specificity were $0.872,0.741$ and 0.809 , respectively (Figure $3 \mathrm{C}$ ). The AUC of the validation group was 0.824 , sensitivity and specificity were 0.833 and 0.619 , respectively (Figure 3D). The extenal validation group of center 2 proved the stability of the model with an AUC, sensitivity and specificity of $0.800,0.729$ and 0.731 , separately (Figure $3 \mathrm{E}$ ). The calibration curve and decision curve showed a good match between the prediction of image model and the real situation (Figure 3A and 3B). All specific parameters about the radiomics model can be found in Table 4. Delong test was found that the classifying efficiency of radiomics was equivalent to that of the senior radiologist ( $P=0.847,0.471$, respectively, Table 5$)$, both of which were higher than that of junior radiologist, and the difference was statistically significant (both, Pcript $>0.05$, Table 5).

Table 4

The specific parameters of radiomics model

\begin{tabular}{|lllllllll|}
\hline & Precision & AUC(95\% $\mathbf{C l})$ & Sensitivity & Specificity & F1-score & Recall & PPV & NPV \\
\hline Train & 0.784 & $0.872(0.789-0.965)$ & 0.741 & 0.809 & 0.714 & 0.741 & 0.690 & 0.844 \\
\hline Validation & 0.762 & $0.824(0.612-1.000)$ & 0.667 & 0.889 & 0.762 & 0.667 & 0.889 & 0.667 \\
\hline Ex-val & 0.727 & $0.800(0.649-0.954)$ & 0.729 & 0.731 & 0.744 & 0.762 & 0.727 & 0.546 \\
\hline Note: EX-val: external validation & & & & & & & \\
\hline AUC: area under the ROC curve & & & & & & & \\
\hline
\end{tabular}


Table 5

The results of Delong test among different models

\begin{tabular}{|lll|}
\hline & Training group & Validation group \\
\hline ER1 vs Radiomics & 0.847 & 0.471 \\
\hline ER2 vs Radiomics & $0.015^{*}$ & $0.002^{\star}$ \\
\hline ER1 vs ER2 & $0.008^{*}$ & $0.042^{*}$ \\
\hline Note: * presents the statistically significant of results \\
\hline
\end{tabular}

\section{Discussion}

In this present study, we found that inflammatory severity in CD can be resolved from MR studies by the radiomics objectively and repeatedly. Moreover, the method has a comparable ability with MaRIA as evaluated by our senior radiologist.

$\mathrm{MRI}$, as a non-invasive method with good soft tissue resolution and no radiation exposure, plays an important role in the management of CD. In this technique, quantitative indicators of inflammation, such as MaRIA was established based on morphological and imaging parameters. However, due to the complexity of intestinal anatomy, high consistency of these features were difficult to guarantee among radiologists. Some studies have reported highly variation in assessing MRI features among radiologists [13,20-21]. In the reading of RCE, agreement was only moderate between senior radiologists (ICC=0.55), and even lower when combined with junior radiologists (ICC=0.42) according to Tielbeek et al [13], which was similar to the results of a recent central reading (the ICC of RCE was 0.59) [21]. Interestingly, the consistency between the two senior radiologists was lower than the four radiologists(including two junior radiologists) as a whole (Kappa coefficient 0.57 vs 0.66 ) in the evaluation of edema parameters, suggesting that subjectivity may have played a role in this evaluation. In terms of of ulcer reading, the consistency reported by current researches are pessimistic, no consistency was detected by Tsai et al [22] and only poor consistency was reported by Rees et al [23]. The results of this study were similar to those previous studies, and no good agreement was obtained in the reading of MRI features between radiologists. Although these MRI imaging features have been standardized to ensure reading consistency among radiologists, subjective and experience differences among radiologists is still an issue, especially among junior radiologists.

Computer-assisted image analysis overcame the subjectivity and improved the reproducibility of findings [24-26]. Radiomic technology allows for a high level of diagnostic accuracy by detecting features smaller than the human eye can discern on MRI. With the addition of machine learning, radiomics technologies have recently garnered strong attention from the scientific community [27-30]. In terms of CD, part of researches [24,31-32] confirmed that it can improve the repeatability and agreement of measurements. Moreover, some previous studies have found correlations between some texture features and the pathological mechanisms of inflammation [33-34]. Therefore, image analysis can be an effective method for inexperienced radiologists to improve the ability to assess CD.

In this study, features with good reproducibility were retained based on MRI, most of which were Wavelet-transformed features, prior studies have suggested that it may reveal heterogeneity in the ROI and suggested a poorer prognosis [35], therefore, it may be an image-based indicator of more severe inflammation or poorer clinical outcome in patients with CD [36]. In addition, "skewness" features based on wavelet transform was retained, a texture analysis study found that skewness is related to angiogenesis [34], a confirmed and important pathological marker of inflammation 
in $\mathrm{CD}$ [37], and Makanyanga et al[33] further confirmed that that the weight of "skewness" feature was related to the enhancement degree of the ROI on MR images, therefore, this type of feature may be an alternation of RCE measured manually by radiologists, which presented only fair consistency in this study between radiologists. However, the specific relationship between retained features and the pathological mechanism of inflammation has not been disclosed in this study and further confirmation is needed.

Finally, we modeled the radiomics by retained features and compared the efficiency of MaRIA. The diagnostic efficacy of the radiomics study were in parallel with senior radiologists who were limited supply, and better than that of junior radiologists. Although subjectivity is also unavoidable when sketching ROI, retained characteristics are highly consistent between radiologists, further preventing the effect of subjectivity. The results of this study suggest that Radiomics could assess the severity of inflammatory steadily and accurately, and has applications in the clinical realm.

There are several limitations of this study. Firstly, our sample size is relatively small in our research center, most subjects preferred clinical follow-up rather than hospitalization, therefore, MRI or endoscopic data may be absent or incomplete. More samples and multicenter study need to be performed. Secondly, we choose the terminal ileum as the ROI, the initial and most involved site of $C D$, not global involvement of the intestinal tract. Thirdly, Since terminal ileum lesions may be discontinuous or asymmetric, accurate point-to-point endoscopic matching is required in the future.

\section{Conclusion}

In summary, while MaRIA is a good criterion for grading CD inflammation, it depends heavily on the experience of the radiologist. The radiomics based on MRI imaging features was objective and reproducible. Our study along this direction has revealed that the radiomics model performs similarly to senior radiologists and may be a important method to assist junior radiologists in assessing severity of inflammation in the terminal ileum.

\section{Declarations}

Ethics approval and consent to participate: The ethics committee of the First Affiliated Hospital of Zhejiang Chinese Medical University approved this retrospective study (2020-KL-035-01) and the need for written informed consent was waived. All methods were performed in accordance with the relevant guidelines and regulations.

Consent for publication: All participants were approved for publication.

Availability of data and materials: The data generated and analyzed during the current study are not publicly available but are available from the corresponding author on reasonable request.

Competing interest: There is no conflict of interest among the authors listed.

\section{Funding}

This work was supported by the Natural Science Foundation of Zhejiang Province (LGD19C040002, LYS19H030001), the Zhejiang Traditional Chinese Medicine Administration (2020ZB091, 2016ZA091) and the Medical Health Science and Technology Project of Zhejiang Provincial Health Commission (2022KY914).

\section{Author Contributions:}


HLD contributed to the study design, data analysis and process, data acquisition, data interpretation, drafting of manuscript; JYL and CG contributed to the data analysis and process; KFJ contributed to the data acquisition and interpretation of endoscopy; HNZ, LJL and HBC contributed to the data acquisition; XNG obtained funding and contributed to the data analysis; HBC and KFZ provided imaging interpretation and evaluation. ZCS obtained funding and contributed to the study design, critical review of manuscript, and study supervision.

Acknowledgments: Not applicable

\section{References}

1. Roda Giulia, Chien Ng Siew, Kotze Paulo Gustavo et al. Crohn's disease.[J].Nat Rev Dis Primers, 2020, 6: 22. DOI: 10.1038/s41572-020-0156-2

2. Clémentine Lauriot dit Prevost, Azahaf M, Nachury M, et al. Bowel damage and disability in Crohn's disease: a prospective study in a tertiary referral centre of the Lémann Index and Inflammatory Bowel Disease Disability Index[J]. Alimentary Pharmacology \& Therapeutics, 2020, 51(9). DOI: 10.1111/apt.15681

3. Rieder F, Fiocchi C, Rogler G. Mechanisms, Management, and Treatment of Fibrosis in Patients With Inflammatory Bowel Diseases[J]. Gastroenterology, 2016, 152(2):340. DOI: 10.1053/j.gastro.2016.09.047

4. Hordonneau C, Buisson A, Scanzi J, et al. Diffusion-Weighted Magnetic Resonance Imaging in Ileocolonic Crohn's Disease: Validation of Quantitative Index of Activity[J]. The American Journal of Gastroenterology, 2013, 109(1). DOI: 10.1038/ajg.2013.385

5. Siddiki H, Fletcher J G, Hara A K, et al. Validation of a lower radiation computed tomography enterography imaging protocol to detect Crohn's disease in the small bowel.[J]. Inflammatory Bowel Diseases, 17(3):778-786. DOI: 10.1002/ibd.21364

6. Steward M J, Punwani S, Proctor I, et al. Non-perforating small bowel Crohn's disease assessed by MRI enterography: Derivation and histopathological validation of an MR-based activity index[J]. European Journal of Radiology, 2012, 81(9):2080-2088. DOI: 10.1016/j.ejrad.2011.07.013

7. Adler J, Punglia D R, Dillman J R, et al. Computed tomography enterography findings correlate with tissue inflammation, not fibrosis in resected small bowel Crohn's disease[J]. Inflammatory Bowel Diseases, 2012, 18(5). DOI: 10.1002/ibd.21801

8. Church P C, Turner D, Feldman B M, et al. Systematic review with meta-analysis: magnetic resonance enterography signs for the detection of inflammation and intestinal damage in Crohn's disease[J]. Alimentary Pharmacology \& Therapeutics, 2014. DOI: 10.1111/apt.13024

9. Takenaka Kento, Ohtsuka Kazuo, Kitazume Yoshio, et al. Utility of Magnetic Resonance Enterography For Small Bowel Endoscopic Healing in Patients With Crohn's Disease.[J].Am J Gastroenterol, 2018, 113: 283-294. DOI: 10.1038/ajg.2017.464

10. Varyani F, Samuel S. "Can MRE replace ileo-colonoscopy for evaluating disease activity in Crohn's Disease?"[J]. Baillière' s Best Practice and Research in Clinical Gastroenterology, 2019, 38-39. DOI: 10.1016/j.bpg.2019.05.008

11. Rimola J, Rodriguez S, Garcia-Bosch 0 , et al. Magnetic resonance for assessment of disease activity and severity in ileocolonic Crohn's disease.[J]. Gut, 2009, 58(8):1113. DOI: 10.1136/gut.2008.167957

12. Rimola J, I Ordás, Rodriguez S, et al. Magnetic resonance imaging for evaluation of Crohn's disease: Validation of parameters of severity and quantitative index of activity[J]. Inflammatory Bowel Diseases, 2011, 17(8):17591768. DOI: $10.1002 /$ ibd.21551

Page $11 / 15$ 
13. Tielbeek Jeroen A W, Makanyanga Jesica C, Bipat Shandra et al. Grading Crohn disease activity with MRI: interobserver variability of MRI features, MRI scoring of severity, and correlation with Crohn disease endoscopic index of severity.[J].AJR Am J Roentgenol, 2013, 201: 1220-8. DOI: 10.2214/AJR.12.10341

14. Fiz F, Vigano L, Nicolò Gennaro, et al. Radiomics of Liver Metastases: A Systematic Review[J]. Cancers, 2020. DOI: $10.3390 /$ cancers 12102881

15. Jiang Y, Wang W, Chen C, et al. Radiomics Signature on Computed Tomography Imaging: Association With Lymph Node Metastasis in Patients With Gastric Cancer[J]. Frontiers in Oncology, 2007, 9. DOI: 10.3389/fonc. 2019.00340

16. Thawani R, Mclane $M$, Beig N, et al. Radiomics and radiogenomics in lung cancer: A review for the clinician[J]. Lung Cancer, 2018, 115:34. DOI: 10.1016/j.lungcan.2017.10.015

17. Mary J Y, Modigliani R, Development and validation of an endoscopic index of the severity for Crohn's disease: a prospective multicentre study. Groupe d'Etudes Thérapeutiques des Affections Inflammatoires du Tube Digestif (GETAID).[J].Gut, 1989, 30: 983-9. DOI: 10.1136/gut.30.7.983

18. I Ordás, Rimola J, Alfaro I, et al. Development and Validation of a Simplified Magnetic Resonance Index of Activity for Crohn's Disease[J]. Gastroenterology, 2019, 157(2):432. DOI: 10.1053/j.gastro.2019.03.051

19. Lichtenstein GR, Loftus EV, Isaacs KL, Regueiro MD, Gerson LB, Sands BE. ACG clinical guideline: management of Crohn's disease in adults[J]. Am J Gastroenterol. 2018;113(4):481-517. DOI: 10.1038/s41395-018-0120-x

20. Ziech M, Bipat S, Roelofs J, et al. Retrospective comparison of magnetic resonance imaging features and histopathology in Crohn's disease patients[J]. European Journal of Radiology, 2011, 80(3):e299-e305. DOI: 10.1016/j.ejrad.2010.12.075

21. Vipul J, Ingrid O, Zou G, et al. Reliability of Measuring Ileo-Colonic Disease Activity in Crohn's Disease by Magnetic Resonance Enterography[J]. Inflammatory Bowel Diseases, 2018(2):2. DOI: 10.1093/ibd/izx040

22. Tsai R, Mintz A, Lin M, et al. Magnetic resonance enterography features of small bowel Crohn's disease activity: an inter-rater reliability study of small bowel active inflammation in clinical practice setting[J]. British Journal of Radiology, 2019, 92(1099):20180930. DOI: 10.1259/bjr.20180930

23. Rees M A, Dillman J R, Anton C G, et al. Inter-radiologist agreement using Society of Abdominal RadiologyAmerican Gastroenterological Association (SAR-AGA) consensus nomenclature for reporting CT and MR enterography in children and young adults with small bowel Crohn disease[J]. Abdominal Radiology, 2019:1-7. DOI: 10.1007/s00261-018-1743-5

24. Puylaert Carl A J, Schüffler Peter J, Naziroglu Robiel E, et al. Semiautomatic Assessment of the Terminal Ileum and Colon in Patients with Crohn Disease Using MRI (the VIGOR++ Project).[J].Acad Radiol, 2018, 25: 10381045. DOI: 10.1016/j.acra.2017.12.024

25. Parmar C, Grossmann P, Rietveld D, et al. Radiomic Machine Learning Classifiers for Prognostic Biomarkers of Head \& Neck Cancer[J]. Frontiers in Oncology, 2015, 5(4). DOI: 10.3389/fonc.2015.00272

26. Yi X, Xiao Q, Zeng F, et al. Computed Tomography Radiomics for Predicting Pathological Grade of Renal Cell Carcinoma[J]. Frontiers in Oncology, 2021, 10:570396. DOI: 10.3389/fonc.2020.570396

27. Y Han, Y Yang, Shi Z S, et al. Distinguishing brain inflammation from grade Il glioma in population without contrast enhancement: a radiomics analysis based on conventional MRI[J]. European Journal of Radiology, 2021, 134(10145):109467. DOI: 10.1016/j.ejrad.2020.109467

28. Arendt C T, Leithner D, Mayerhoefer M E, et al. Radiomics of high-resolution computed tomography for the differentiation between cholesteatoma and middle ear inflammation: effects of post-reconstruction methods in a dual-center study[J]. European Radiology, 2021:1-8. DOI: 10.1007/s00330-020-07564-4

Page 12/15 
29. Andre E, Brett K, Novoa R A, et al. Dermatologist-level classification of skin cancer with deep neural networks. [J].Nature, 2017, 542: 115-118. DOI: 10.1038/nature21056

30. Diagnostic Assessment of Deep Learning Algorithms for Detection of Lymph Node Metastases in Women With Breast Cancer[J]. JAMA: The Journal of the American Medical Association, 2017, 318(22):2199-.31. DOI: 10.1001/jama.2017.14585

31. Stidham R W, Binu E, Waljee A K, et al. Assessing Small Bowel Stricturing and Morphology in Crohn's Disease Using Semi-automated Image Analysis[J]. Inflammatory Bowel Diseases, 2019(5):5. DOI: 10.1093/ibd/izz196

32. Naziroglu R E, Puylaert C, Tielbeek J, et al. Semi-automatic bowel wall thickness measurements on MR enterography in patients with Crohn's disease[J]. British Journal of Radiology, 2017:20160654. DOI: 10.1259/bjr.20160654

33. Makanyanga J, Ganeshan B, Rodriguez-Justo M, et al. MRI texture analysis (MRTA) of T2-weighted images in Crohn's disease may provide information on histological and MRI disease activity in patients undergoing ileal resection[J]. European Radiology, 2017, 27(2):589-597

34. Bhatnagar G, Makanyanga J, Ganeshan B, et al. MRI texture analysis parameters of contrast-enhanced T1weighted images of Crohn's disease differ according to the presence or absence of histological markers of hypoxia and angiogenesis[J]. Abdominal Radiology, 2016, 41(7):1261-1269. DOI: 10.1007/s00261-016-0657-3

35. Depeursinge A, Foncubierta-Rodriguez A, Ville D, et al. Three-dimensional solid texture analysis in biomedical imaging: Review and opportunities[J]. Medical Image Analysis, 2014, 18(1):176-196. DOI:

10.1016/j.media.2013.10.005

36. Lichtenstein G R, Loftus E V, Isaacs K L, et al. ACG Clinical Guideline: Management of Crohn's Disease in Adults[J]. Official journal of the American College of Gastroenterology | ACG, 2018, 113. DOI:

10.1038/ajg.2018.27

37. Danese S, Sans M, Motte C, et al. Angiogenesis as a Novel Component of Inflammatory Bowel Disease Pathogenesis[J]. Gastroenterology, 2006, 130(7):2060-2073. DOI: 10.1053/j.gastro.2006.03.054

\section{Figures}




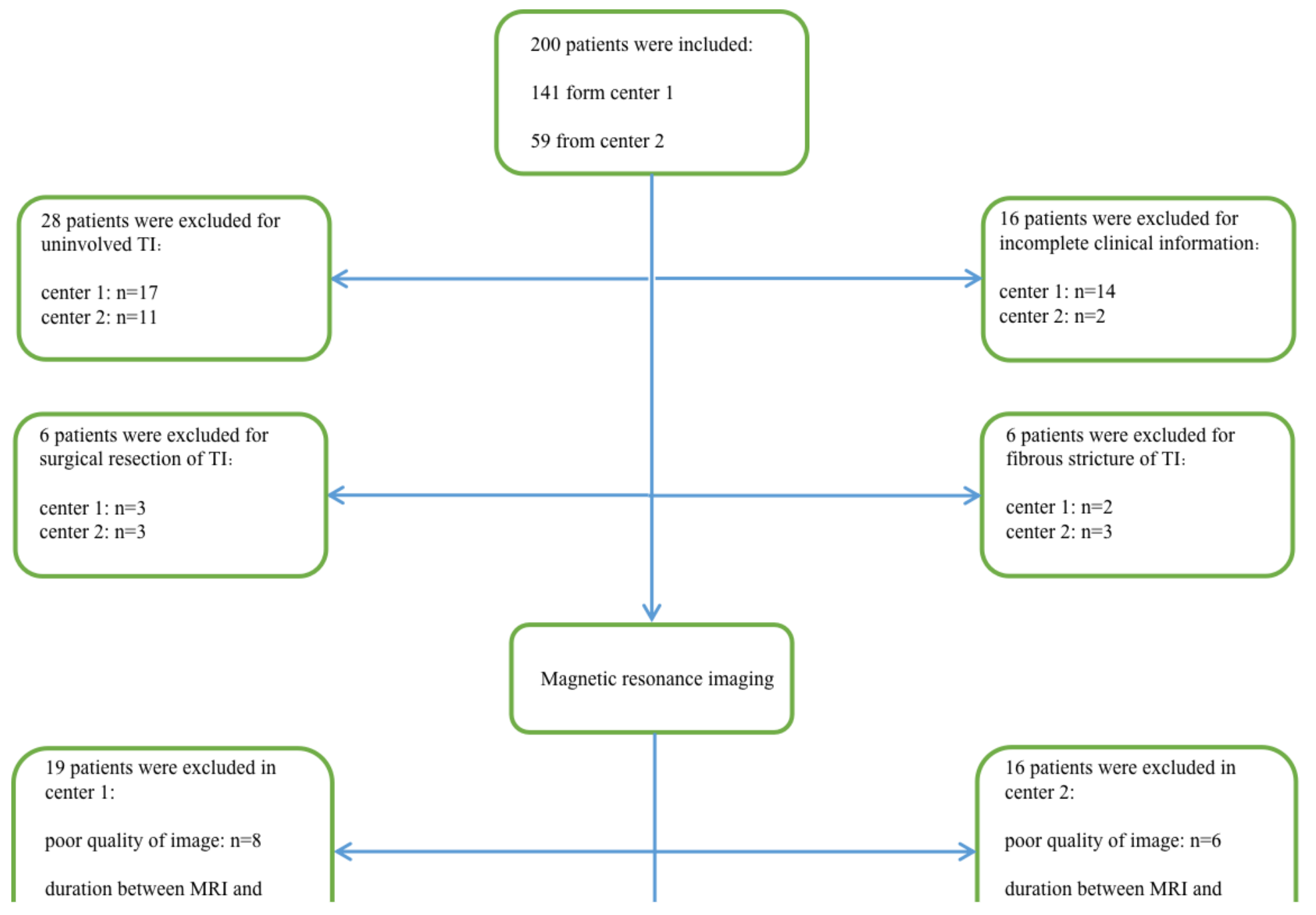

\section{Figure 1}

Flow diagram of the study subjects. 121 patients were included according to the included and excluded criteria. The included patients were examined by MRI and endoscopy, and had complete clinical information needed for the study.

\section{Figure 2}

The workflow of Radiomics analysis procedures. A represent the outline of non-active group, a 34-year-old male diagnosed A2B1L1 according to Montreal classification, with a tCDEIS of 4.5凶B presents the outline of active group, a 65-year-old woman diagnosed A3B2L3 according to Montreal classification, with a tCDEIS of 7.5.

Note: GLCM: Gray Level Co-occurrence Matrix 
GLRLM: Gray Level Run Length Matrix

GLSZM: Gray Level SizeZone Matrix,

GLDM: Gray Level Dependence Matrix

NGTDM: Neighborhood Gray-Tone Difference Matrix

\section{Figure 3}

The output of Radiomics model in assessing the inflammatory severity of $\mathrm{TI}(\mathrm{A}-\mathrm{E})$. A presents the reliability curve; $\mathrm{B}$ presents the clinical decision curve; $\mathrm{C}$ represents the result of training group. $\mathrm{D}$ displays the result of validation, and $\mathrm{E}$ shows the results of the external validation group. 